\title{
Multiple genotype-phenotype association study reveals intronic variant pair on SIDT2 associated with metabolic syndrome in a Korean population
}

\author{
Sanghoon Moon ${ }^{1 \dagger}$, Young Lee ${ }^{1,2+}$, Sungho Won ${ }^{3}$ and Juyoung Lee ${ }^{1 *}$
}

\begin{abstract}
Background: Metabolic syndrome is a risk factor for type 2 diabetes and cardiovascular disease. We identified common genetic variants that alter the risk for metabolic syndrome in the Korean population. To isolate these variants, we conducted a multiple-genotype and multiple-phenotype genome-wide association analysis using the family-based quasi-likelihood score (MFQLS) test. For this analysis, we used 7211 and 2838 genotyped study subjects for discovery and replication, respectively. We also performed a multiple-genotype and multiple-phenotype analysis of a gene-based single-nucleotide polymorphism (SNP) set.
\end{abstract}

Results: We found an association between metabolic syndrome and an intronic SNP pair, rs7107152 and rs1242229, in SIDT2 gene at 11q23.3. Both SNPs correlate with the expression of SIDT2 and TAGLN, whose products promote insulin secretion and lipid metabolism, respectively. This SNP pair showed statistical significance at the replication stage.

Conclusions: Our findings provide insight into an underlying mechanism that contributes to metabolic syndrome.

Keywords: Multiple variants, Multiple traits, Metabolic syndrome, 11q23.3, SIDT2

\section{Introduction}

Metabolic syndrome is a cluster of metabolic risk factors for cardiovascular disease and type 2 diabetes that are attributable to both genetic and environmental factors [13]. The National Cholesterol Education Program's Adult Treatment Panel III report (2001) defined metabolic syndrome as a combination of components such as high blood pressure, elevated fasting plasma glucose, high serum triglycerides, and abnormal low-density lipoprotein (LDL) and high-density lipoprotein (HDL) cholesterol levels [4]. Because of the fast-growing economy and rapid industrialization of Korea, metabolic syndrome is likely to become a major public health problem [5].

Many genetic variants that are associated with metabolic syndrome have been identified by genome-wide association studies (GWASs). However, because these known

\footnotetext{
* Correspondence: jylee@cdc.go.kr

†Sanghoon Moon and Young Lee contributed equally to this work. ${ }^{1}$ Division of Genome Research, Center for Genome Science, Korea National Institute of Health, Cheongju, Chungcheongbuk-do 28159, South Korea
} Full list of author information is available at the end of the article genetic variants account for only a fraction of the heritability of metabolic syndrome, the genetic determinants of this condition remain undefined [6, 7]. Many common variants with very small effect sizes that are widely distributed across the genome cannot be identified by the use of traditional GWAS cutoffs [8]. Experimental sample sizes must be large because the statistical power to detect associations between DNA variants and a trait depends on the sample size [9]. However, the cost and difficulties of sample collection inhibit the ability to continuously increase the sample size of GWASs [10]. Joint analysis approaches that analyze multiple genotypes and phenotypes have shown improved ability to detect variants relative to single-variant association analyses of the same-size sample [11-13]. A statistical approach called the MFQLS (http://healthstat.snu.ac.kr/software/mfqls/) test enables the estimation of the genetic relation matrix from population-based samples [14]. From the results of a single test for association with a set of traits, multiple genotype-multiple phenotype analysis reduces the number of 
tests and mitigates the multiple testing issues, resulting in increased statistical power [13, 14]. This approach identifies genetic variants that have pleiotropic effects for metabolic syndrome and other diseases.

We conducted a multiple single nucleotide polymorphism (SNP)-multiple trait analysis to identify genetic variants associated with metabolic syndrome by utilizing 10,049 samples from Korean subjects. After the multiplegenotype and multiple-phenotype analysis (multi-SNPmulti-trait analysis), 27 SNP pairs were associated with metabolic syndrome in the discovery stage and successfully replicated. Of those pairs, from the joint analysis of a single genotype and multiple phenotypes (single-SNPmulti-trait analysis), we found that three SNP pairs in the respective genes $S I D T 2, U B A S H 3 B$, and CUX2 were significant in the multi-SNP-multi-trait analysis but not significant in the single-SNP-multi-trait analysis. SIDT2 was not previously reported in the NHGRI-EBI GWAS Catalog (https://www.ebi.ac.uk/gwas/) [15] and was significant in the gene-based SNP set and multi-trait analysis. The Genotype-Tissue Expression (GTEx) database shows rs7107152 and rs1242229 on SIDT2 correlates with SIDT2 and TAGLN expression (https://www.gtexportal.org/ home/) [16]. Our findings support the effectiveness of the multi-SNP-multi-trait analysis to identify new susceptible loci in complex diseases.

\section{Results}

\section{Multi-SNP-multi-trait analysis}

We conducted the multi-SNP-multi-trait genome-wide analysis of metabolic syndrome using 10,049 samples from Korean subjects. We considered six quantitative components of metabolic syndrome: systolic blood pressure (SBP), diastolic blood pressure (DBP), high-density lipoprotein (HDL), fasting plasma glucose (FPG), triglyceride, and waist circumference. Through the multi-SNP-multitrait genome-wide analysis, adjusted for age and sex, 27 SNP pairs satisfied a Bonferroni-adjusted $P$ value threshold of $P<0.05\left(P=1.45 \times 10^{-7}\right)$ and were successfully replicated (Tables 1 and 2). All but four of the mapped genes (SIK3, SIDT2, UBASH3B, and CUX2) had been previously reported to be associated with metabolic syndrome, as indicated by a keyword search of "metabolic syndrome" in the GWAS catalog (Tables 1 and 2).

\section{Single SNP set-multi-trait analysis}

The relation of the individual SNPs, including those on SIK3, SIDT2, UBASH3B, and CUX2, to metabolic syndrome was further examined by single-SNP-multi-trait analysis, adjusted for age and sex. Intronic SNPs on SIK3 showed genome-wide significance in single-SNP-multitrait analysis (Table 1), but SNPs on SIDT2, UBASH3B, and CUX2 not identified by this approach (Table 2).

\section{Gene-based SNP set-multi-trait analysis}

We conducted gene-based SNP set-multi-trait analysis on 14,475 SNP sets. The mean and median numbers of SNPs in a set were 6.895 and 3 , respectively (Additional file 1 : Table S1). Table 3 shows the gene-based test results. Three genes reached a Bonferroni-adjusted $P$ value threshold $\left(P<3.45 \times 10^{-6}\right)$. All three genes satisfied statistical significance in the meta-analysis. The number of SNPs in three genes ranges from 3 to 14 . SNP sets in two genes (PAFAH1B2 and SIDT2) showed significant $P$ values of $<$ $3.45 \times 10^{-6}$ in the gene-based test and reached a nominal $P$ value threshold of $<0.05$ in the replication stage. Although the results of tests in which 14 SNPs were utilized in CUX2 were significant in the discovery stage, there was only a suggested $P$ value of 0.06 in the replication analysis (Table 3). Table 3 also shows the SNPs used in each SNP set.

\section{Expression QTL pattern of identified SNP pair}

To examine the correlation between two SNPs (rs7107152 and rs1242229) on the SIDT2 gene and gene expression, we utilized three online resources: the Genotype-Tissue Expression (GTEx) project (https://www.gtexportal.org/ home/) [16], the NESDA NTR Conditional Expression Quantitative Trait Loci (eQTL) catalog (https://eqtl.onder zoek.io/), and RegulomeDB (http://www.regulomedb.org/) [17]. All three databases showed that the two SNPs correlated with SIDT2 and TAGLN expressions. The GTEx project showed that for rs7107152, the statistical significance of SIDT2 and TAGLN expression in whole blood was $P=3.89 \times 10^{-14}$ and $P=3.59 \times 10^{-47}$, respectively (Additional file 1: Figure S1A), and for rs1242229, the $P$ values were $3.64 \times 10^{-13}$ and $4.78 \times 10^{-12}$, respectively (Additional file 1: Figure S1B) [16]. Additional file 1: Figure $\mathrm{S} 1 \mathrm{C}-\mathrm{F}$ show gene expression patterns by genotype for the two SNPs. The eQTL catalog showed similar gene expression patterns for the two SNPs (Additional file 1: Figure S2). The SNP rs1242229 was mainly expressed in SIDT2 and TAGLN, whereas the SNP rs7107152 showed gene expression patterns not only in SIDT2 and TAGLN but also in PCSK7 and PAFAH1B2 (Additional file 1: Figure S2). Additional file 1: Figure S3 shows the gene expression pattern for rs1242229 in RegulomeDB [17].

\section{Correlation test with previously reported SNPs}

To calculate the correlation of the identified SNPs (rs7107152 and rs1242229) in the current study and nearby SNPs reported in the GWAS catalog (intronic variant rs530885291, associated with HDL cholesterol level, and intergenic variant rs508487, associated with triglyceride level), we conducted a pairwise linkage disequilibrium (LD) analysis utilizing the SNP Annotation and Proxy Search (SNAP) server (https://www.broadinstitute.org/ snap/snap) [18]. The pairwise LD analysis showed that 


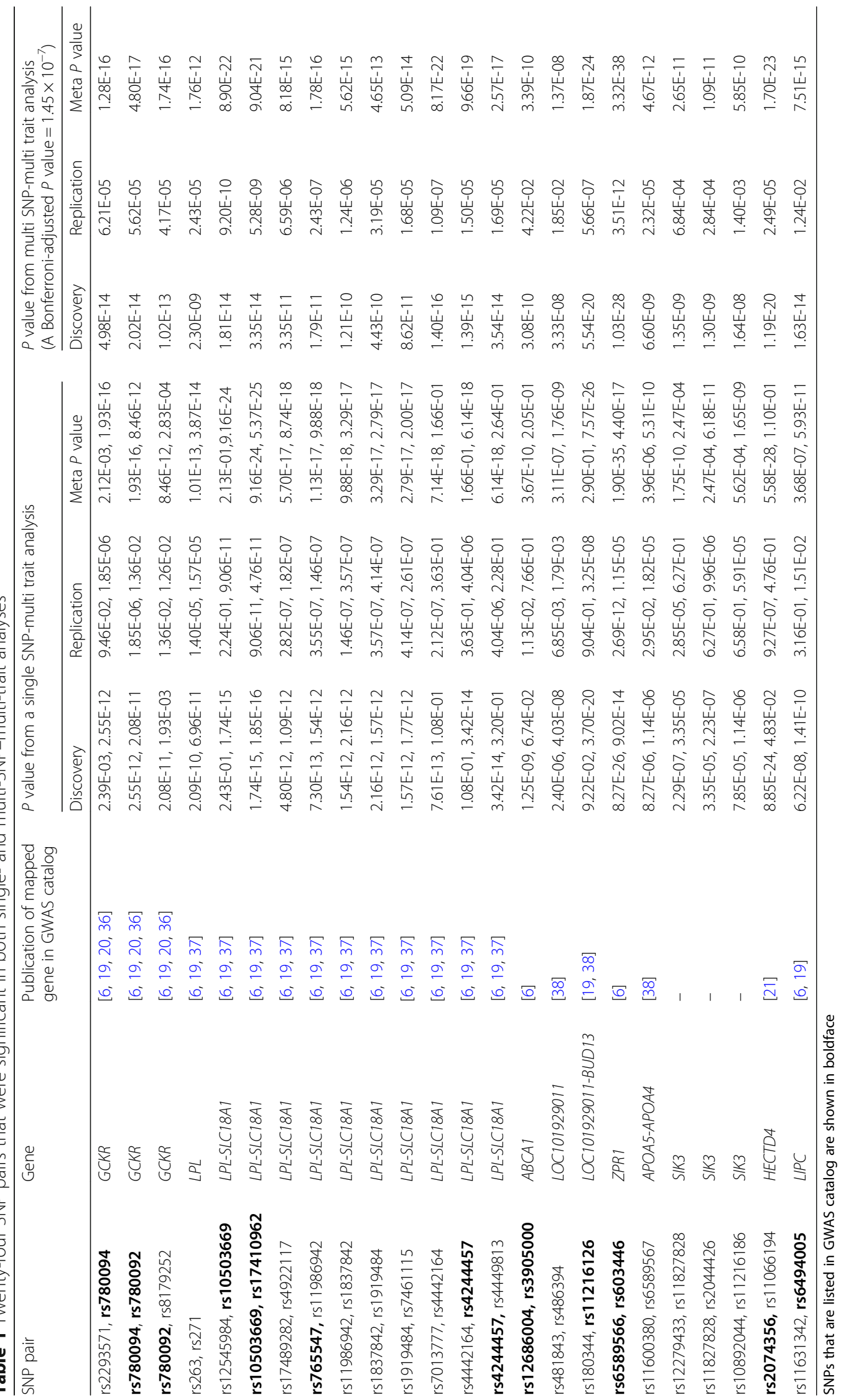


Table 2 Three SNP pairs that were significant only in the multi-SNP-multi-trait analysis

\begin{tabular}{|c|c|c|c|c|c|c|c|c|c|c|}
\hline \multirow[t]{2}{*}{ SNP pair } & \multirow[t]{2}{*}{$\mathrm{CHR}$} & \multirow[t]{2}{*}{$\begin{array}{l}\text { Position } \\
\text { (hg19) }\end{array}$} & \multirow[t]{2}{*}{ Gene } & \multirow[t]{2}{*}{ Annotation } & \multicolumn{3}{|c|}{ Single-multi $P$ value } & \multicolumn{3}{|c|}{$\begin{array}{l}\text { Multi-multi } P \text { value } \\
(\text { A Bonferroni-adjusted } P \text { value of discovery } \\
\text { stage }=1.45 \times 10^{-7} \text { ) }\end{array}$} \\
\hline & & & & & Discovery & Replication & Meta $P$ value & Discovery & Replication & Meta $P$ value \\
\hline rs7107152 & 11 & $117,056,080$ & SIDT2 & Intronic & $2.59 \mathrm{E}-01$ & $8.43 \mathrm{E}-01$ & $5.51 \mathrm{E}-01$ & 1.40E-08 & $2.32 \mathrm{E}-03$ & $8.17 \mathrm{E}-10$ \\
\hline rs1242229 & 11 & $117,062,370$ & SIDT2 & Intronic & 5.39E-03 & $7.68 \mathrm{E}-02$ & $3.64 \mathrm{E}-03$ & & & \\
\hline rs10892876 & 11 & $122,540,281$ & UBASH3B & Intronic & 2.93E-01 & $1.27 \mathrm{E}-01$ & $1.60 \mathrm{E}-01$ & $3.34 \mathrm{E}-12$ & $3.82 \mathrm{E}-03$ & $4.21 \mathrm{E}-13$ \\
\hline rs12290043 & 11 & $122,540,528$ & UBASH3B & Intronic & $1.62 \mathrm{E}-01$ & $1.46 \mathrm{E}-01$ & $1.12 \mathrm{E}-01$ & & & \\
\hline rs886126 & 12 & $111,679,214$ & CUX2 & Intronic & $3.51 \mathrm{E}-01$ & $1.79 \mathrm{E}-01$ & 2.37E-01 & $5.09 \mathrm{E}-13$ & 3.35E-03 & $5.97 \mathrm{E}-14$ \\
\hline rs2078851 & 12 & $111,690,579$ & CUX2 & Intronic & 7.31E-05 & $6.63 \mathrm{E}-02$ & $6.42 \mathrm{E}-05$ & & & \\
\hline
\end{tabular}

Single-multi $P$ value $P$ value from a single-SNP-multi-trait association analysis, multi-multi $P$ value $P$ value from a multi-SNP-multi-trait association analysis, $C H R$ chromosome, Meta $P$ value $P$ value from meta-analysis

rs1242229 had a squared correlation of $r^{2}=0.167$ with rs508487 (Fig. 1). The correlation score between rs7107152 and rs530885291, however, was lower than the cutoff score.

\section{SNP pair-single trait analysis}

A radar chart shows the results of the rs7107152 and rs1242229 SNP metabolic syndrome-component trait association pair analysis (Additional file 1: Figure S4). The SNP pair correlated with HDL $\left(P=5.87 \times 10^{-5}\right)$ and triglycerides $\left(P=1.67 \times 10^{-9}\right)$. Moreover, it showed a suggestive association with diastolic blood pressure $(P=0.07)$. But it did not correlate with waist circumference $(P=0.75)$, fasting plasma glucose $(P=0.55)$, systolic blood pressure $(P=0.29)$.

\section{Discussion}

Utilizing the MFQLS test, we found 27 SNP pairs associated with metabolic syndrome based on multi-SNPmultiple continuous phenotypes. Our keyword search

Table 3 Results from a gene-based SNP set and multi-trait analysis

\begin{tabular}{|c|c|c|c|c|c|c|c|}
\hline \multirow[t]{2}{*}{ Gene } & \multirow[t]{2}{*}{$\begin{array}{l}\text { The number } \\
\text { of SNP }\end{array}$} & \multirow[t]{2}{*}{ Chromosome } & \multirow[t]{2}{*}{$\begin{array}{l}\text { Position } \\
\text { (hg19) }\end{array}$} & \multirow[t]{2}{*}{ Maximum $r^{2}$} & \multicolumn{3}{|c|}{$\begin{array}{l}P \text { value } \\
\text { (a Bonferroni-adjusted } P \text { value of discovery stage } P=3.45 \times 10^{-6} \text { ) }\end{array}$} \\
\hline & & & & & Discovery & Replication & Meta $P$ value \\
\hline \multirow[t]{3}{*}{ PAFAHIB2 } & rs12420127 & 11 & $117,035,319$ & 0.466 & $4.00 \mathrm{E}-07$ & 3.99E-03 & $3.39 \mathrm{E}-08$ \\
\hline & rs10790175 & & $117,034,729$ & & & & \\
\hline & rs10892082 & & $117,039,325$ & & & & \\
\hline \multirow[t]{4}{*}{ SIDT2 } & rs2269399 & 11 & $117,066,353$ & 0.456 & $2.31 \mathrm{E}-07$ & $3.83 \mathrm{E}-03$ & $1.93 \mathrm{E}-08$ \\
\hline & rs1242229 & & $117,062,370$ & & & & \\
\hline & rs1784042 & & $117,065,476$ & & & & \\
\hline & rs7107152 & & $117,056,080$ & & & & \\
\hline \multirow[t]{14}{*}{ CUX2 } & rs7952972 & 12 & $111,646,519$ & 0.561 & $3.28 \mathrm{E}-10$ & $6.34 \mathrm{E}-02$ & $5.32 \mathrm{E}-10$ \\
\hline & rs886126 & & $111,679,214$ & & & & \\
\hline & rs7300082 & & $111,737,115$ & & & & \\
\hline & rs4766553 & & $111,634,281$ & & & & \\
\hline & rs1265566 & & $111,716,376$ & & & & \\
\hline & rs9783423 & & $111,639,456$ & & & & \\
\hline & rs7398833 & & $111,786,892$ & & & & \\
\hline & rs16941414 & & $111,779,792$ & & & & \\
\hline & rs6489979 & & $111,614,736$ & & & & \\
\hline & rs16941284 & & $111,610,723$ & & & & \\
\hline & rs16941319 & & $111,646,853$ & & & & \\
\hline & rs11065851 & & $111,723,739$ & & & & \\
\hline & rs756825 & & $111,598,202$ & & & & \\
\hline & rs7300860 & & $111,754,597$ & & & & \\
\hline
\end{tabular}




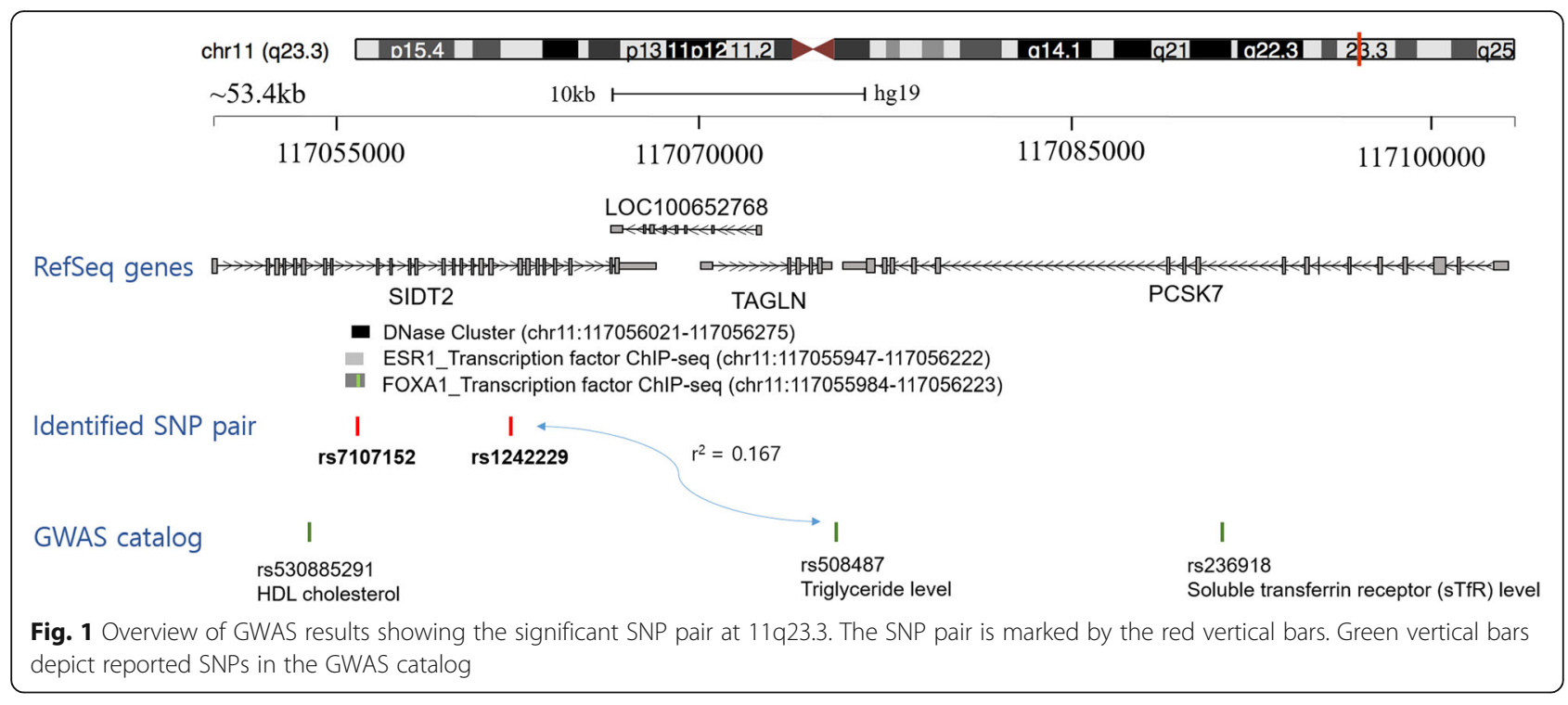

for the term "metabolic syndrome" found single-variant association results in the GWAS catalog. The association of metabolic syndrome or metabolic syndrome-component traits with 21 SNP pairs was found in the GWAS catalog [15], whereas the association of 6 SNP pairs on the genes SIK3, SIDT2, UBASH3B, and CUX2 was not found (Table 1). Not only lipid loci but also insulin-associated loci associated with metabolic syndrome. However, most of the variants identified were present in known lipid loci. In contrast, a relatively small number of variants were in other metabolic syndrome-component traits. For example, genetic variants such as rs780092 and rs780094 mapped on the GCKR gene were susceptible variants relative to total cholesterol, fasting glucose level, and lipid metabolism phenotypes $[6,19,20]$. An SNP from the pairs identified here, rs2074356, which mapped on the HECTD4 gene, was previously associated with the glycemic trait [21]. Six of the SNP pairs identified have shown an association with the lipid trait-associated LD region spanning BUD13-ZNF259, APOA5-A4-C3-A1, and SIK3 [6, 13, 19, 22]. Povel et al. [22] reported a systematic review of genetic variants and metabolic syndrome. They suggested that although disturbances in metabolic syndrome-component traits have been proposed to activate metabolic syndrome, most SNPs associated with metabolic syndrome are in genes involved in lipid metabolism [23]. Moreover, Kristiansson et al. [6] showed that genes from the lipid metabolism pathway are factors in metabolic syndrome. However, these investigators found little evidence associated with other metabolic syndrome-component traits such as hypertension and glucose intolerance [6]. Our results were consistent with previous studies.

Through the single-SNP-multi-trait analysis, we identified four SNP pairs that were associated with metabolic syndrome but were not in the GWAS catalog. Intronic SNPs on the SIK3 gene showed genome-wide significance in the single-SNP-multi-trait analysis (Table 1). SNPs on the $U B A S H 3 B$ and $C U X 2$ genes could not be identified by single-SNP-multi-trait analysis (Table 2), whereas the association of $U B A S H 3 B$ and $C U X 2$ with metabolic syndrome-component traits was reported in the GWAS catalog. For example, two variants, rs7128198 on the 5 -untranslated region and rs7941030 upstream of $U B A S H 3 B$, were associated with total cholesterol and HDL level, respectively [24, 25]. The intergenic variant rs12229654 between MYL2 and CUX2 showed a pleiotropic effect associated with metabolic syndrome, HDL, and glycemic traits [21, 26]. An association between cardiovascular disease and rs886126, which also identified in our study, was previously reported [27].

Utilizing multi-SNP-multi-trait analysis, we found an association between the SIDT2 gene and metabolic syndrome or metabolic syndrome-component traits based on an association between metabolic syndrome and rs7107152 and rs1242229. The SNP rs7107152 is within the DNase cluster in the region of transcription factors such as ESR1 and FOXA1 (Fig. 1). The SNPs rs530885291 and rs508487 showed in the GWAS catalog as proximal to rs7107152 and rs1242229, respectively [15]. However, the pairwise LD score between rs1242229 and rs508487 was low $\left(r^{2}=0.167\right)$, indicating little correlation between these two SNPs.

We performed another multi-SNP-multi-trait analysis based on the SNP set test (gene-based test). SNP sets on the PAFAH1B2, SIDT2, and CUX2 genes showed significant $P$ values in the gene-based test (a Bonferroni-adjusted $P$ value threshold is $P=3.45 \times 10^{-6}$ ). PAFAH1B2 and SIDT2 reached a replication $P$ value of $<0.05$. The SNP set on CUX2 suggested a $P$ value of 0.06 in the 
replication stage (Table 3); a larger sample size might have resulted in a significant $P$ value. The SNP set rs7107152 and rs1242229 on SIDT2 was also significant, supporting the association of SIDT2 with metabolic syndrome.

We found evidence from the three online databases that rs7107152 and rs1242229, which are in an intron of SIDT2, correlated with the expression of SIDT2 and TAGLN (Additional file 1: Figure S1). Two recent eQTL studies provide further evidence that these two SNPs alter gene expression that is relevant to metabolic syndrome-component traits $[28,29]$. From the eQTL analysis between SNP and whole-blood gene expression in 5,257 Framingham Heart Study participants, rs1242229 was reported as a proxy SNP for altered SIDT2 and TAGLN expression in triglyceride [28]. Huan et al. [29] investigated cis and trans eQTL by utilization of the human whole-blood transcriptome data from Framingham Heart Study pedigrees. The SNP rs7107152 also altered SIDT2 expression in triglyceride [8]. The radar chart shows that these variants increase HDL and triglyceride levels among metabolic syndrome-component traits, supporting the hypothesis that genes are a key factor in the link between lipid metabolism and metabolic syndrome (Additional file 1: Figure S4) [6, 22]. We conferred that lipid traits such as HDL and TG have the greatest impact on metabolic syndrome, but weak associations such as DBP may also have an impact. However, due to the limitations of current research, the potential weak association must interpret carefully. The eQTL SNPs correlated with SIDT2 and TAGLN expression enriched in the 100-kb region $(117000-117,100 \mathrm{~kb})$ around SIDT2 and TAGLN (Additional file 1: Figure S5).

SID1 transmembrane family member 2 (SIDT2) is a lysosomal integral membrane protein that promotes insulin secretion [30]. Recently, Gao et al. [31] described its activity in insulin secretion. Sidt $2^{-/-}$mice exhibit weight loss and increased fasting glucose levels and impaired glucose tolerance. These investigators identified mouse SIDT2 function in lipid metabolism. SIDT2-deficient mice have increased serum triglyceride [32]. TAGLN (Transgelin, $\operatorname{sm} 22 \alpha)$ is an actin-binding protein expressed in smooth muscle cells [33]. Yang et al. [34] revealed that the most enriched pathways caused by SM22 $\alpha$ knockout in mice were lipid metabolism, inflammation, and hematopoiesis. We hypothesize that the SNP pair associated with metabolic syndrome activate expression of the genes SIDT2 and TAGLN. A difference in gene expression might inhibit insulin secretion, lipid metabolism, and adipogenesis, resulting in metabolic syndrome (Additional file 1: Figure S6). Although evidence supports the association between the identified SNPs and metabolic syndrome, functional investigations of the SNPs are needed. However, functional investigation using identified 27 SNP pairs in non-coding regions is limited. Given additional information such as imputed SNPs, a metabolic syndrome-associated genetic variant in coding regions may be detected by the statistical test used in the present study.

\section{Conclusions}

Our results show that a multi-SNP-multi-trait analysis is an efficient approach for finding variants that have not previously been isolated from single-variant-multi-trait analysis. The advantage of this approach is an increase in statistical power that results from considering the combined effects of two variants. Because $P$ values of identified variants such as rs7107152 and rs1242229 did not reach statistical significance, these variants cannot be identified by single-variant analysis of the same sample size. Although previously identified variants are in lipid loci, these variants and their mapped genes are not reported in metabolic syndrome. Our findings provide insight into the genetic variant contribution to metabolic syndrome.

\section{Methods}

\section{Study design and participants}

To identify susceptible variant sets associated with metabolic syndrome, we conducted a multi-SNP-multi-trait analysis through the two stages: discovery and replication analysis (Fig. 2). For the discovery stage, we used genome data from the Ansan/Ansung cohort in the Korean Genome Epidemiology Study, which is known as the Korea Association REsource (KARE) project [35]. In the subsequent replication stage, we used the Health Examinees (HEXA) study cohort, data from which were also used in the GWASs [26, 27]. All participants were between 40 and 69 years of age. Informed consent was obtained from all participants. This study was approved by the ethical committee of the Korea Centers for Disease Control and Prevention Institutional Review Board. Detailed demographic information of participants is shown in Table 4.

\section{Quality control in GWAS}

The KARE data consisted of 10,004 samples genotyped with Affymetrix Genome-Wide Human SNP array 5.0 $[26,35]$. We selected 8,842 individuals genotyped with 352,228 SNPs from the quality control process. The quality control criteria and process have been described [21, 26, 35]. We used 351,983 SNPs for the multi-SNPmulti-trait analysis after exclusion of 245 SNPs with ambiguous chromosome numbers and positions. We selected 7,211 of 8,842 participants who did not take lipid-lowering or anti-diabetes medication. For the replication analysis, we selected 2,838 of 3,701 participants from the HEXA study after those taking medication were excluded. 


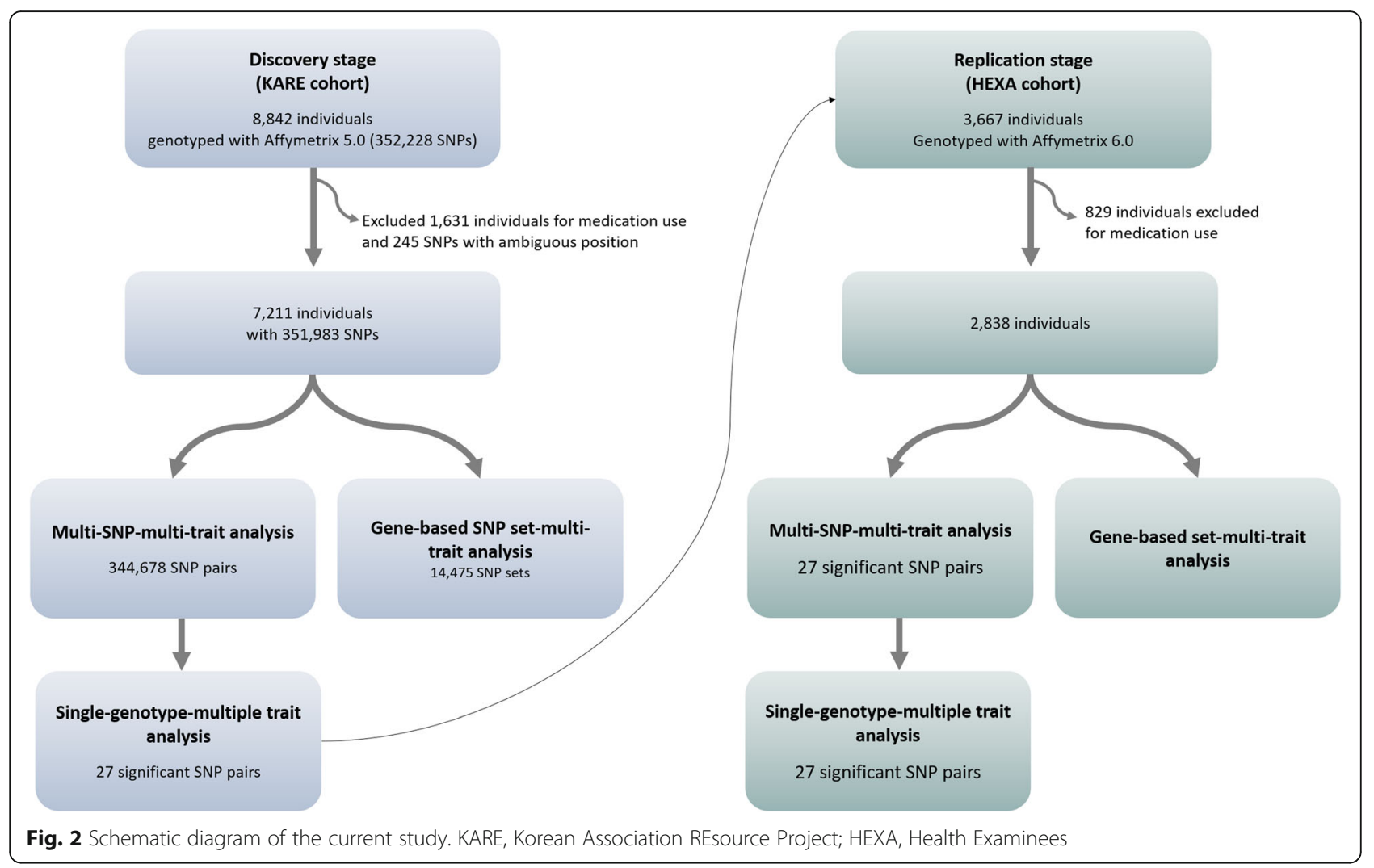

\section{Phenotyping}

We considered six quantitative traits as components of metabolic syndrome. We used the National Cholesterol Education Program Expert Panel on Detection, Evaluation, and Treatment of High Blood Cholesterol in Adults (Adult Treatment Panel III) final report guideline as metabolic syndrome definition [4]. For waist circumference, we used modified Asian guidelines, which reduce the limit from $>$ $102 \mathrm{~cm}$ to $>90 \mathrm{~cm}$ for men and from $>88 \mathrm{~cm}$ to $>80 \mathrm{~cm}$ for women. Thus, a participant was considered to have metabolic syndrome if he or she had three of the five following features: (1) triglyceride $>150 \mathrm{mg} / \mathrm{dL}$, (2) $\mathrm{HDL}<$ $40 \mathrm{mg} / \mathrm{dL}$ for men and $<50 \mathrm{mg} / \mathrm{dL}$ for women, (3) waist circumference $>90 \mathrm{~cm}$ for men and $>80 \mathrm{~cm}$ for women, (4) fasting plasma glucose $>110 \mathrm{mg} / \mathrm{dL}$, and (5) blood pressure threshold $>130 \mathrm{mmHg}(\mathrm{SBP})$ and $>85 \mathrm{mmHg}(\mathrm{DBP})$.

\section{Multiple SNP set-multiple trait association analysis}

Previously, Won et al. [14] proposed a statistical method for the joint analysis of multiple phenotypes and

Table 4 Characteristics of the study participants

\begin{tabular}{|c|c|c|c|c|c|}
\hline & \multicolumn{3}{|l|}{ KARE } & \multicolumn{2}{|l|}{ HEXA } \\
\hline & Case $(n=1328)$ & Control $(n=5870)$ & Not determined $(n=13)$ & Case $(n=309)$ & Control $(n=2529)$ \\
\hline Age & $53.9(8.65)$ & $50.52(8.62)$ & $61.31(8.02)$ & $54(7.92)$ & $51.48(7.88)$ \\
\hline $\operatorname{Sex}(m / f)$ & $561 / 767$ & $3000 / 2870$ & $4 / 9$ & $184 / 125$ & 1039/1490 \\
\hline SBP & $128.15(18.13)$ & $112.36(15.52)$ & $128.36(16.07)$ & $132.19(13.77)$ & $118.68(13.47)$ \\
\hline $\mathrm{DBP}$ & $81.97(11.46)$ & $72.32(10.49)$ & $74.92(6.43)$ & $83.54(9.12)$ & $75.39(9.47)$ \\
\hline FPG & $94(29.82)$ & $84.31(14.79)$ & $80.67(1.53)$ & $101.49(25.38)$ & $90.15(22.3)$ \\
\hline Triglyceride & $241.35(134.62)$ & $138.94(83.58)$ & $180.92(122.1)$ & $224.38(134.91)$ & $106.35(74.49)$ \\
\hline log triglyceride & $5.38(0.44)$ & $4.82(0.44)$ & $5.04(0.55)$ & $5.28(0.5)$ & $4.52(0.52)$ \\
\hline $\mathrm{HDL}$ & $38.35(6.78)$ & $46.54(10.06)$ & $42.58(8.39)$ & $43.5(8.88)$ & $57(13.16)$ \\
\hline WC & $90.33(7.01)$ & $79.98(7.82)$ & $87.27(7.97)$ & $90.69(6.86)$ & $80.44(8.13)$ \\
\hline
\end{tabular}

$n$ sample size; SBP systolic blood pressure, DBP diastolic blood pressure, FPG fasting plasma glucose, HDL high-density lipoprotein, WC waist circumference KARE Korean Association Resource Project, HEXA Health Examinee cohort

Data are shown as the mean (SD) 
genotypes. This method, the MFQLS (http://healthstat.snu.ac.kr/software/mfqls/), can be utilized for both quantitative and dichotomous phenotypes. It can be applied to large-scale, genome-wide association analysis as well as family-based samples. The empirical power test showed that it is statistically more efficient than existing methods. In addition, the genome-wide association analysis of 1,801 individuals with obesity showed that $P$ values from the MFQLS were markedly less than those from other methods [14]. In our study, two types of multiple SNP sets, such as paired SNPs and gene-based SNPs having more than three variants, were considered to be multiple genotypes. For the multi-SNP-multi-trait analysis, the MFQLS incorporates both multiple traits and SNPs into a single test statistic. For example, given two traits and two SNPs, the MFQLS tests $H_{0}: \beta_{11}=\beta_{12}=\beta_{21}=\beta_{22}=0$, where $\beta_{i j}$ denotes the effect of association between the $i$ th SNP and the $j t h$ trait. The effect of these genotypes and the statistical significance of this effect are greater when multiple genotypes and phenotypes are correlated. Of 351,983 SNPs, 344,677 SNP pairs with MAF >0.01 were selected (a Bonferroni-adjusted $P$ value threshold, $P=$ $\left.1.45 \times 10^{-7}\right)$. Additional file 1: Table S2 shows MAF of identified SNPs. We extended the gene-based genome-wide association analysis with multiple traits. To select an SNP set for gene-based analysis, we included common SNPs in the first set on the platform used in the discovery and replication stages. We selected gene-based tag SNPs captured with $r^{2} \geq 0.8$ by the use of Tagger (http://www.broad.mit.edu/mpg/tagger/) for the second set. Consequently, 14,475 SNP sets were selected for gene-based analysis (a Bonferroni-adjusted $P$ value threshold, $P=3.45 \times 10^{-6}$ ). Table 3 and Additional file 1: Table S1 show information about the SNP sets used for the gene-based test.

Two-stage analyses such as estimation of correlation between each SNP and calculation of statistics are used to run the MFQLS.

The Fisher's combined probability test was used to calculate the meta-analysis from discovery and replication results by application of MADAM in the R package.

\section{Single-SNP-multi-trait association analysis}

To determine whether a significant SNP pair identified in the multi-SNP-multi-trait analysis was still significant if a different approach was used, we performed a joint analysis between single genotype and multiple phenotypes. An SNP and multiple traits were incorporated into the statistics of a single test and three traits were given, $H_{0}: \beta_{1}=\beta_{2}$ $=\beta_{3}=0$ and $H_{1}$ : not $H_{0}$, where $\beta_{i}$ denotes the effect of association between a SNP and the ith trait. Covariates such as age and sex were adjusted in the analysis.
SNP pair-metabolic syndrome-component trait association analysis

To determine which metabolic syndrome-component traits are related to the SNP pair rs7107152 and rs1242229, we performed an association analysis between the SNP pair and each metabolic syndrome-component trait.

\section{Additional analyses from online data resources}

To examine the expression pattern of each SNP of an identified SNP pair, we utilized three online data resources that provide eQTL information: the portal for GTEx [16], RegulomeDB [17], and the NESDA NTR Conditional eQTL Catalog. SNAP was applied to calculate the correlation between identified SNPs and previously known GWAS SNPs [18].

\section{Additional files}

Additional file 1: Supplementary information. Figure S1. Screenshot of GTEx database for significant SNP pair rs7107152 and rs1242229. Figure S2. Screenshot of NESDA NTR Conditional eQTL Catalog for rs7107152 and rs1242229. Figure S3. Screenshot of RegulomeDB for rs1242229. Figure S4. Radar chart showing the result of the rs7107152 and rs1242229 SNP pair-single trait (metabolic syndrome-component trait) association analysis. Figure $\mathbf{S 5}$. Screenshot from the GTEx database of the eQTL SNP-enriched region around SIDT2 and TAGLN

(chr11:117,000,000-117,100,000). Figure S6 Model for how significant SNP pair rs7107152/rs1242229 may affect metabolic syndrome risk. Table S1. Characteristics of gene-based SNPs set. Table S2. Minor allele frequency of identified SNPs. (PDF $975 \mathrm{~kb}$ )

Additional file 2: MFQLS results. (TXT $16795 \mathrm{~kb}$ )

\section{Abbreviations}

DBP: Diastolic blood pressure; eQTL: Expression quantitative trait loci; FPG: Fasting plasma glucose; GTEx: Genotype-tissue expression; GWAS: Genome-wide association study; HDL: High-density lipoprotein cholesterol; HEXA: Health Examinees; KARE: Korean Association REsource Project; LDL: Low-density lipoprotein cholesterol; MFQLS: The family-based quasi-likelihood score test; SBP: Systolic blood pressure; SIDT2: SID1 transmembrane family member 2; TAGLN: Transgelin; TC: Total cholesterol; TG: Triglyceride

\section{Funding}

This work was funded by intramural grants from the Korean National Institute of Health (2013-NG73002-00, 2016-NI73005-00) and supported by the Basic Science Research Program through the National Research Foundation of Korea Grant funded by the Korean Government (NRF-2014S1A2A2028559). Data were also provided by the Korean Genome Analysis Project (4845-301) and the National Biobank of Korea, which were supported by the Korea Center for Disease Control and Prevention, Republic of Korea.

\section{Availability of data and materials}

The data supporting the findings of this study is included within the Additional file 2

\section{Author's contributions}

SM contributed to the analysis and interpretation of data and to the drafting of the manuscript. YL contributed to the design of the study, analysis of the data, and drafting of the manuscript. SW contributed to the revision of the manuscript. $J$ contributed to the concept and design of the study and to the revision of the manuscript. All authors approved submission of the final version of the article for publication. 


\section{Ethics approval and consent to participate}

All procedures were in accordance with the ethical standards of the responsible committee on human experimentation (Approved IRB number of Korea Centers for Disease Control and Prevention in Korea: 2013-01CON-19-P). Informed written consent was obtained from all patients.

\section{Consent for publication}

All patients consented to the publication of their data.

\section{Competing interests}

The authors declare that they have no competing interests.

\section{Publisher's Note}

Springer Nature remains neutral with regard to jurisdictional claims in published maps and institutional affiliations.

\section{Author details}

'Division of Genome Research, Center for Genome Science, Korea National Institute of Health, Cheongju, Chungcheongbuk-do 28159, South Korea. ${ }^{2}$ Veterans Medical Research Institute, Veterans Health Service Medical Center, Seoul 05368, South Korea. ${ }^{3}$ Department of Public Health Science, Seoul National University, Seoul 08826, South Korea.

\section{Received: 5 April 2018 Accepted: 8 October 2018}

\section{Published online: 01 November 2018}

\section{References}

1. Ordovas JM, Shen J. Gene-environment interactions and susceptibility to metabolic syndrome and other chronic diseases. J Periodontol. 2008;79: 1508-13.

2. Brown $A E$, Walker M. Genetics of insulin resistance and the metabolic syndrome. Curr Cardiol Rep. 2016;18:75.

3. Dragsbaek K, Neergaard JS, Laursen JM, Hansen HB, Christiansen C, Beck-Nielsen $\mathrm{H}$, Karsdal MA, Brix S, Henriksen K. Metabolic syndrome and subsequent risk of type 2 diabetes and cardiovascular disease in elderly women: challenging the current definition. Medicine (Baltimore). 2016;95:e4806.

4. Third Report of the National Cholesterol Education Program (NCEP). Expert panel on detection, evaluation, and treatment of high blood cholesterol in adults (adult treatment panel III) final report. Circulation. 2002;106:3143-421.

5. Hong AR, Lim S. Clinical characteristics of metabolic syndrome in Korea, and its comparison with other Asian countries. J Diabetes Investig. 2015;6:508-15.

6. Kristiansson K, Perola M, Tikkanen E, Kettunen J, Surakka I, Havulinna AS, Stancakova A, Barnes C, Widen E, Kajantie E, Eriksson JG, Viikari J, Kahonen $\mathrm{M}$, et al. Genome-wide screen for metabolic syndrome susceptibility Loci reveals strong lipid gene contribution but no evidence for common genetic basis for clustering of metabolic syndrome traits. Circ Cardiovasc GenetCirc Cardiovasc Genet. 2012:5:242-9.

7. Abou Ziki MD, Mani A. Metabolic syndrome: genetic insights into disease pathogenesis. Curr Opin Lipidol. 2016;27:162-71.

8. Pare G, Asma S, Deng WQ. Contribution of large region joint associations to complex traits genetics. PLoS Genet. 2015;11:e1005103.

9. Klein RJ. Power analysis for genome-wide association studies. BMC Genet. 2007:8:58

10. Spencer CC, Su Z, Donnelly P, Marchini J. Designing genome-wide association studies: sample size, power, imputation, and the choice of genotyping chip. PLoS Genet. 2009;5:e1000477.

11. Klei L, Luca D, Devlin B, Roeder K. Pleiotropy and principal components of heritability combine to increase power for association analysis. Genet Epidemiol. 2008:32:9-19.

12. Stephens M. A unified framework for association analysis with multiple related phenotypes. PLoS One. 2013;8:e65245.

13. Zhou X, Stephens M. Efficient multivariate linear mixed model algorithms for genome-wide association studies. Nat Methods. 2014;11:407-9.

14. Won S, Kim W, Lee S, Lee Y, Sung J, Park T. Family-based association analysis: a fast and efficient method of multivariate association analysis with multiple variants. BMC Bioinformatics. 2015;16:46

15. MacArthur J, Bowler E, Cerezo M, Gil L, Hall P, Hastings E, Junkins H, McMahon A, Milano A, Morales J, Pendlington ZM, Welter D, Burdett T, et al. The new NHGRI-EBI catalog of published genome-wide association studies (GWAS catalog). Nucleic Acids Res. 2017;45:D896-901.
16. GTEx consortium. Genetic effects on gene expression across human tissues. Nature. 2017:550:204-13.

17. Boyle AP, Hong EL, Hariharan M, Cheng Y, Schaub MA, Kasowski M, Karczewski KJ, Park J, Hitz BC, Weng S, Cherry JM, Snyder M. Annotation of functional variation in personal genomes using RegulomeDB. Genome Res. 2012;22:1790-7.

18. Johnson AD, Handsaker RE, Pulit SL, Nizzari MM, O'Donnell CJ, de Bakker PI. SNAP: a web-based tool for identification and annotation of proxy SNPS using HapMap. Bioinformatics. 2008;24:2938-9.

19. Kraja AT, Vaidya D, Pankow JS, Goodarzi MO, Assimes TL, Kullo IJ, Sovio U, Mathias RA, Sun W, Franceschini N, Absher D, Li G, Zhang Q, et al. A bivariate genome-wide approach to metabolic syndrome: STAMPEED consortium. Diabetes. 2011:60:1329-39.

20. Setoh K, Terao C, Muro S, Kawaguchi T, Tabara Y, Takahashi M, Nakayama T, Kosugi S, Sekine A, Yamada R, Mishima M, Matsuda F. Three missense variants of metabolic syndrome-related genes are associated with alpha-1 antitrypsin levels. Nat Commun. 2015;6:7754

21. Go MJ, Hwang JY, Kim YJ, Hee OJ, Heon Kwak S, Soo Park K, Lee J, Kim BJ, Han BG, Cho MC, Cho YS, Lee JY. New susceptibility loci in MYL2, C12orf51 and OAS1 associated with 1-h plasma glucose as predisposing risk factors for type 2 diabetes in the Korean population. J Hum Genet. 2013;58:362-5.

22. Povel CM, Boer JMA, Reiling E, Feskens EJM. Genetic variants and the metabolic syndrome: a systemic review. Obes Rev. 2011;12:952-67.

23. Braun TR, Been LF, Singhal A, Worsham J, Ralhan S, Wander GS, Chambers JC, Kooner JS, Aston CE, Sanghera DK. A replication study of GWAS-derived lipid genes in Asian Indians: the chromosomal region 11q23.3 harbors loci contributing to triglycerides. PLoS One. 2012;7:e37056.

24. Willer CJ, Schmidt EM, Sengupta S, Peloso GM, Gustafsson S, Kanoni S, Ganna A, Chen J, Buchkovich ML, Mora S, Beckmann JS, Bragg-Gresham JL, Chang HY, et al. Discovery and refinement of loci associated with lipid levels. Nat Genet. 2013;45:1274-83.

25. Teslovich TM, Musunuru K, Smith AV, Edmondson AC, Stylianou IM, Koseki M, Pirruccello JP, Ripatti S, Chasman DI, Willer CJ, Johansen CT, Fouchier SW, Isaacs A, et al. Biological, clinical and population relevance of 95 loci for blood lipids. Nature. 2010;466:707-13.

26. Kim YJ, Go MJ, Hu C, Hong CB, Kim YK, Lee JY, Hwang JY, Oh JH, Kim DJ, Kim NH, Kim S, Hong EJ, Kim JH, et al. Large-scale genome-wide association studies in East Asians identify new genetic loci influencing metabolic traits. Nat Genet. 2011;43:990-5.

27. Lee JY, Lee BS, Shin DJ, Woo Park K, Shin YA, Joong Kim K, Heo L, Young Lee J, Kyoung Kim Y, Jin Kim Y, Bum Hong C, Lee SH, Yoon D, et al. A genome-wide association study of a coronary artery disease risk variant. J Hum Genet. 2013:58:120-6.

28. Yao C, Chen BH, Joehanes R, Otlu B, Zhang X, Liu C, Huan T, Tastan O, Cupples LA, Meigs JB, Fox CS, Freedman JE, Courchesne $P$, et al. Integromic analysis of genetic variation and gene expression identifies networks for cardiovascular disease phenotypes. Circulation. 2015:131:536-49.

29. Huan $T$, Liu C, Joehanes $R$, Zhang $X$, Chen BH, Johnson AD, Yao C, Courchesne P, O'Donnell CJ, Munson PJ, Levy D. A systematic heritability analysis of the human whole blood transcriptome. Hum Genet. 2015;134: 343-58.

30. Jialin G, Xuefan G, Huiwen Z. SID1 transmembrane family, member 2 (Sidt2): a novel lysosomal membrane protein. Biochem Biophys Res Commun. 2010; 402:588-94.

31. Gao J, Yu C, Xiong Q, Zhang Y, Wang L. Lysosomal integral membrane protein Sidt2 plays a vital role in insulin secretion. Int J Clin Exp Pathol. 2015;8:15622-31.

32. Gao J, Zhang Y, Yu C, Tan F, Wang L. Spontaneous nonalcoholic fatty liver disease and ER stress in Sidt2 deficiency mice. Biochem Biophys Res Commun. 2016:476:326-32

33. Sayar N, Karahan G, Konu O, Bozkurt B, Bozdogan O, Yulug IG. Transgelin gene is frequently downregulated by promoter DNA hypermethylation in breast cancer. Clin Epigenetics. 2015;7:104

34. Yang M, Jiang H, Li L. Sm22alpha transcription occurs at the early onset of the cardiovascular system and the intron 1 is dispensable for its transcription in smooth muscle cells during mouse development. Int $」$ Physiol Pathophysiol Pharmacol. 2010;2:12-9.

35. Cho YS, Go MJ, Kim YJ, Heo JY, Oh JH, Ban HJ, Yoon D, Lee MH, Kim DJ, Park M, Cha SH, Kim JW, Han BG, et al. A large-scale genome-wide association study of Asian populations uncovers genetic factors influencing eight quantitative traits. Nat Genet. 2009;41:527-34. 
36. Ridker PM, Pare G, Parker A, Zee RY, Danik JS, Buring JE, Kwiatkowski D, Cook NR, Miletich JP, Chasman DI. Loci related to metabolic-syndrome pathways including LEPR, HNF1A, IL6R, and GCKR associate with plasma Creactive protein: the Women's Genome Health Study. Am J Hum Genet. 2008;82:1185-92

37. Zabaneh D, Balding DJ. A genome-wide association study of the metabolic syndrome in Indian Asian men. PLoS One. 2010;5:e11961.

38. Zhu Y, Zhang D, Zhou D, Li Z, Fang L, Yang M, Shan Z, Li H, Chen J, Zhou X, Ye W, Yu S, Cai L, et al. Susceptibility loci for metabolic syndrome and metabolic components identified in Han Chinese: a multi-stage genomewide association study. J Cell Mol Med. 2017;21:1106-16.

Ready to submit your research? Choose BMC and benefit from:

- fast, convenient online submission

- thorough peer review by experienced researchers in your field

- rapid publication on acceptance

- support for research data, including large and complex data types

- gold Open Access which fosters wider collaboration and increased citations

- maximum visibility for your research: over $100 \mathrm{M}$ website views per year

At $\mathrm{BMC}$, research is always in progress.

Learn more biomedcentral.com/submissions 